\title{
A multi-channel EMG-driven FES solution for stroke rehabilitation
}

\author{
Yu Zhou ${ }^{1}$, Yinfeng Fang ${ }^{2}$, Jia Zeng ${ }^{1}$, Kairu $\mathrm{Li}^{2}$, and Honghai Liu ${ }^{1}$ \\ ${ }^{1}$ State Key Laboratory of Mechanical System and Vibration, \\ Shanghai Jiao Tong University, Shanghai, China \\ $\{$ hnllyu, jia.zeng, honghai.liu\}@sjtu.edu.cn, \\ http://bbl.sjtu.edu.cn/ \\ ${ }^{2}$ Group of Intelligent System and Biomedical Robotics, \\ School of Creative Technologies, University of Portsmouth \\ Portsmouth, United Kingdom \\ \{yinfeng.fang, kairu.li\}@port.ac.uk
}

\begin{abstract}
Functional electrical stimulation(FES) has been applied to stroke rehabilitation for many years. However, users are usually involved in open-loop fixed cycle FES systems in clinical, which is easy to cause muscle fatigue and reduce rehabilitation efficacy. This paper proposes a multi-surface EMG-driven FES integration solution for enhancing upper-limb stroke rehabilitation. This wireless portable system consists of sEMG data acquisition module and FES module, the former is used to capture sEMG signals, the latter of multi-channel FES output can be driven by the sEMG. Preliminary experiments proved that the system has outperformed existing similar systems and that sEMG can be effectively employed to achieve different FES intensity, demonstrating the potential for active stroke rehabilitation.
\end{abstract}

Keywords: Functional electrical stimulation (FES), surface electromyography (sEMG), integration system, stroke rehabilitation.

\section{Introduction}

FES uses short electrical pulses in specific motor neurons to generate contractions in paralyzed muscles[1]. Since first used for foot drop rehabilitation by Liberson in 1960[2], FES has become one of the important and effective treatments for stroke rehabilitation [3,4]. Most FES systems used in clinical stroke rehabilitation settings are passive open-loop control systems, which output the stimulation current at a predefined fixed model. Thus, it is easy to induce muscle fatigue and requires the users' constant attention to operate them. Moreover, it excludes the active participation of subjects and decreases the neuromuscular activity and energy energy consumption $[5,6]$. The rehabilitation efficiency can be further improved when FES is applied with the EMG feedback, in which patients voluntary movement intention can be detected and stimulation process is adjusted by the muscle state in real time. Thus, the muscle fatigue is alleviated because of the lower average stimulation intensity compared to the cycling 
fixed FES. The EMG-driven closed-loop FES has been studied and shows an advantage over the cycling FES[7-9].

There still exist problems: 1) The use of separate FES and EMG devices to form the closed loop not only faces the communication and real-time problems between the subsystems but also makes the whole system cumbersome and timeconsuming to attach and remove, which becomes one of the main reasons why FES/EMG closed-loop control strategies have not been widely used in clinical applications[1]. 2) Most existing commercial FES systems, such as the ParaStep system (Sigmedics. USA), the RehaStim2 (HASOMED GmbH, Germany) and the NESS H200 (Bioness, USA), are open-loop. Some other devices, such as the Compex Motion stimulator (Compex SA, Swiss), the WalkAide foot drop stimulator (NeuroMotion, USA), the NESS L300 (BIONESS, USA), are equipped with sensor interfaces for force-sensitive-resistors (FSR), accelerometers or push buttons to determine when the stimulation is required according to the users' physical state. However, the sensor input of them performs more like an on/off trigger which does not represent or make full use of users' movement intention or muscle status; Thus, an integration EMG-driven rehabilitation system is desired to solve the problems above.

This study proposes an attempt of a wireless multichannel EMG/FES integration solution for upper-limb stroke rehabilitation. The sEMG from subjects can be used to trigger the FES module, and as an index measuring to which degree a stroke patient recovered.

\section{Multi-channel EMG/FES integration solution}

This section presents the overall design of the EMG/FES integration system. As shown in Fig. 1, the overall architecture is composed of seven parts: electronic switch module (ESM), signal acquisition module (SAM), microcontroller unit (MCU), electric stimulator output module (ESOM), power supply module (PSM), bluetooth module (BM) and graphical user interface (GUI). The channels work in a time-sharing way, which means they are used to capture EMG signals in signal sampling model and output FES current in electrical stimulation model. The ESM, controlled by MCU I/O ports, is used to switch the connection between electrodes-to-ESOM and electrodes-to-SAM, and so that to protect SAM from the heavy current damage from ESOM. The EMG-driven FES is based on the signal sampling model and the electrical stimulation model. In the signal sampling model, the analog raw signals are amplified, low-pass filtered and digitized by SAM and then delivered to MCU for further processing, after that the digital signals can be wirelessly transmitted to the GUI. In the electric stimulation model, the MCU makes ESOM output the stimulation current in a certain parameter configuration (such as amplitude, frequency and pulse width) according to the EMG processing results. 


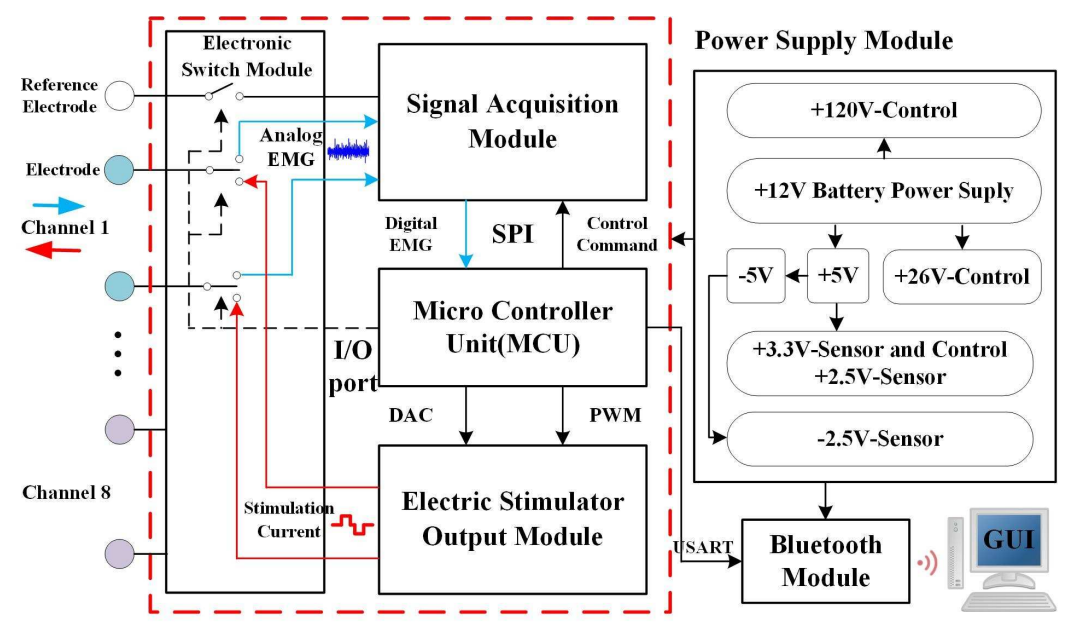

Fig. 1. The architecture of the sEMG/FES integration system.

\section{1 sEMG Acquisition Module}

The raw sEMG signals are always accompanied with various kinds of noises, such as physiological noise, ambient noise (50 or $60 \mathrm{~Hz}$, power line radiation), electrochemical noise from the skin-electrode interface and so on. So our previous study makes use of instrumentation amplifier to suppress common mode noise, band pass filter based on operational amplifiers to extract valid frequency band of EMG signal $(20 \mathrm{~Hz}-500 \mathrm{~Hz})$ and remove baseline noise as well as movement artifact, and comb filter to suppress the $50 \mathrm{~Hz}$ power line noise and multiples thereof. After all those filtering processes, the signals are digitized by the analogto-digital converter (ADC) in MCU[10].

In this study, we chose the ADC integrated chip ADS1299 (Texas Instruments) as the main component of SAM. As shown in Fig. 2, the raw EMG signals are amplified, sampled and low-pass filtered in ADS1299 (the sampling rate is $1 \mathrm{kHz}$ ). Then the digitized sEMG signals are transmitted into MCU (STM32F103VCT6, STMicroelectronics) through the serial peripheral interface (SPI). The comb filter (center frequency, $50 \mathrm{~Hz}$ and multiples thereof) and highpass filter (cut-off frequency, $20 \mathrm{~Hz}$ ) towards the signals are realized in MCU to remove the baseline noise and movement artifact.

The low-pass filter is a on-chip digital third-order sinc filter whose Z-domain transfer function is (1). The Z-domain transfer function of the comb filter is (2). The high-pass filter is a six-order Butterworth filter defined by (3) in Z-domain. Compared to the design in[10], the hardware design in this study can not only extract clean sEMG but also save much space in PCB design.

$$
H(Z)_{\sin c}=\left|\frac{1-Z^{-N}}{1-Z^{-1}}\right|^{3}
$$




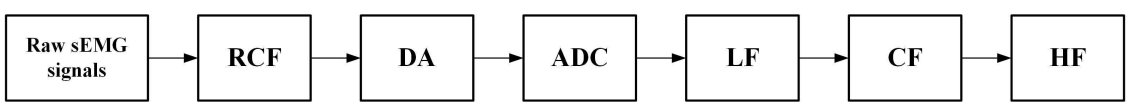

Fig. 2. The sEMG signal processing flow. RCF is the RC filter. DA, ADC and LF are the differential amplification, analog-to-digital conversion and low-pass filter within ADS1299. CF and HF are the comb filter and high-pass filter respectively in MCU.

where $N$ is the decimation ratio of the filter.

$$
H(Z)_{c o m b}=\frac{i-1}{i}-\frac{1}{i} Z^{-T}-\frac{1}{i} Z^{-2 T}-\cdots-\frac{1}{i} Z^{-i T}
$$

where $T$ is a constant determined by sampling frequency $(1 \mathrm{kHz})$ and the basic frequency of the power line noises $(50 \mathrm{~Hz}) ; i$ is the filter order, which determines the length of the previous signals being used to estimate noise.

$$
H(Z)_{b u t t e r w o r t h}=\frac{\sum_{k=0}^{M} b_{k} Z^{-k}}{1-\sum_{l=1}^{N} a_{l} Z^{-l}}
$$

where $a$ and $b$ are coefficients; $N$ and $M$ are determined by the filter order, meaning the length of the previous signals to be used.

\section{$2.2 \quad$ Functional Electric Stimulation Module}

The main parts of the functional electric stimulation module are the constantcurrent source circuit (Fig. 3a) and the bridge circuit (Fig. 3b). The magnitude of the stimulation current can be calculated by (4).

$$
I_{d}=\frac{R_{2} U_{R E F}}{\left(R_{2}+R_{3}\right) R_{s}}
$$

where $I_{d}$ is the stimulation current applied to skin, $U_{R E F}$ is the amplified DAC output from $\mathrm{MCU}$, and $R_{s}$ is the sampling resistance to capture the stimulation magnitude of current in real time (shown in Fig. 3a). The current magnitude is the feedback to the circuit to maintain the constant current.

Under the control of the PWM (Pulse-Width Modulation) signals from the timers in MCU, the bridge circuit can realize the biphasic square wave stimulation current with desired frequency and duration. No matter symmetric biphasic pulses or asymmetric biphasic pulses, the area of the negative phase should be equal to that of the positive phase. The negative phase plays an important role in eliminating the charge accumulation in the skin and avoiding tissue damage. In order to avoid the two constant-current sources working at the same time, which will make the stimulation current into confusion, a short interval time $(100 \mu \mathrm{s})$ is set between PWM1 and PWM2, which results in an interval between the positive and negative phase. 


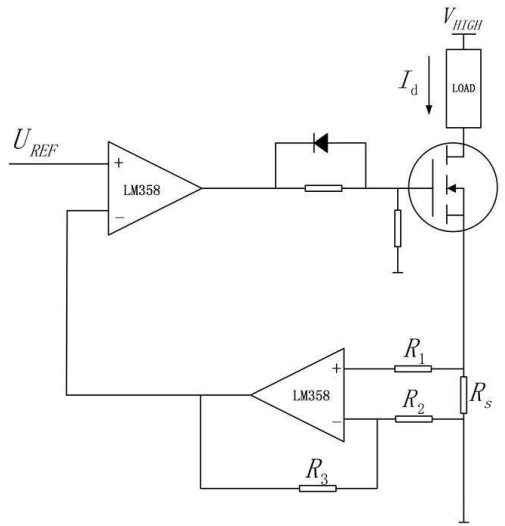

(a)

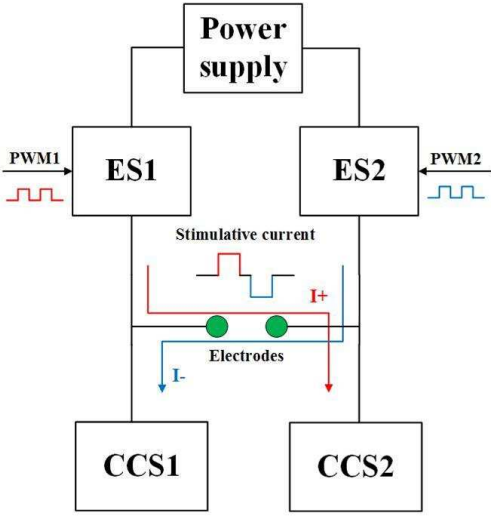

(b)

Fig. 3. The main functional part of the functional electric stimulation module: (a) Electrical schematic representation of constant-current source; (b) Schematic representation of the bridge circuit in ESOM. ES and CCS are electrical switch and constant-current source respectively. The positive phase current I+ is generated when the CCS 2 works and the negative phase current I- is generated when the CCS 1 works.

\section{System evaluation and experiments}

The function of sEMG acquisition, FES output and EMG-driven FES were evaluated respectively. It should be noted that all the experiment procedures in this study were approved by the SJTU School Ethics Committee and all subjects gave written informed consent and provided permission for publication of photographs for scientific and educational purposes.

\subsection{EMG Acquisition Evaluation}

In this part, two commercial sEMG sensors DataLOGMWX8 (Biometrics Ltd, UK) and Tringo Wireless (Delsys Inc, USA) were involved to be compared with the integration system with respect to the time-frequency domain characteristics. A healthy subject was asked to hold on hand close and hand open for $5 \mathrm{~s}$ in turn at a moderate level of effort according to the instructions on a computer screen, and the sEMG was recorded at the same time. The protocol was repeated on the same subject for testing different devices. And the electrodes/sensors were placed on the same belly position of flexor carpi ulnaris (FCU) after cleaning with alcohol[11]. As shown in Fig. 4a, the EMG/FES system could well detect EMG signals between $20 \mathrm{~Hz}$ and $350 \mathrm{~Hz}$ and showed better performance of the suppression for $50 \mathrm{~Hz}$ and multiples thereof than the two commercial devices.

In order to evaluate the signal quality of the integration system, signal-noise ratio (SNR) is compared among the integration system and the commercial devices mentioned before. A healthy subject without nerve and limb disease took 
part in the experiment. During one trial, the subject was asked to hold on the grip force sensor and keep the grip force at the level of 50\% maximum volunteer contraction (MVC) with the vision feedback from computer for $10 \mathrm{~s}$ and then relax for $10 \mathrm{~s}$, meanwhile the sEMG signals were detected with the electrodes/sensors placed on the belly position of FCU. The protocol was repeated on the same subject for testing different devices. The SNR was calculated by equation (5). And the result is shown in Fig. 4b. It indicates that the signal quality of the integration system is better than the two commercial devices.
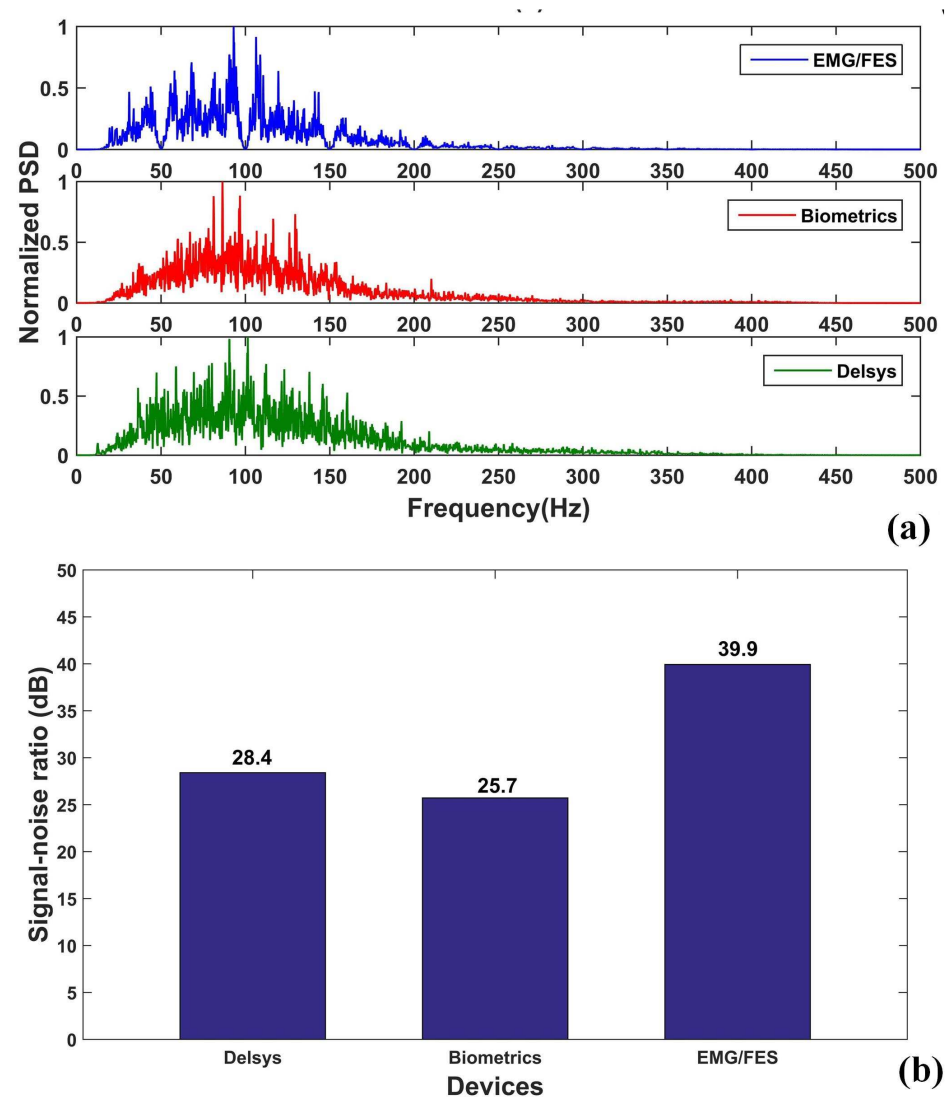

Fig. 4. Signals comparison between the proposed EMG/FES system and commecial sEMG acquisition systems in: (a) Normalized PSD of the acquired sEMG signals (b) Signal-noise ratio.

$$
S N R=20 \log \frac{R M S_{\text {signal }}}{R M S_{\text {noise }}}
$$




\subsection{FES Output Evaluation}

The parameters of the stimulation current consist of amplitude, pulse width and frequency. Fig. 5 shows the waveform of the stimulation current generated by the proposed system with different parameters. It indicates that the system can output normal symmetrical and asymmetrical bipolar square wave for stimulation according to the design, though there is still small fluctuation appearing upon the positive phase when generating currents with certain amplitudes, which may cause uncomfortable acanthesthesia when applied to human skin.

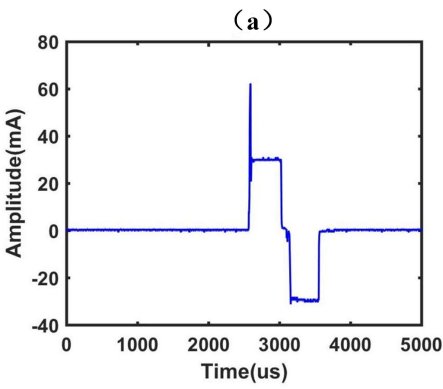

(c)

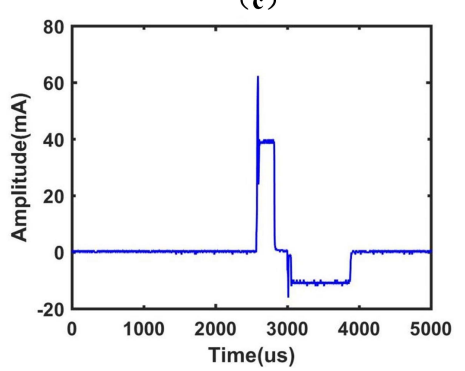

(b)

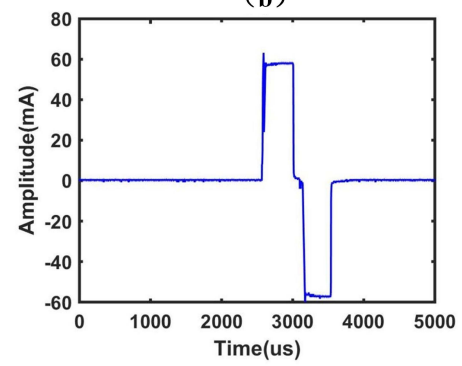

(d)

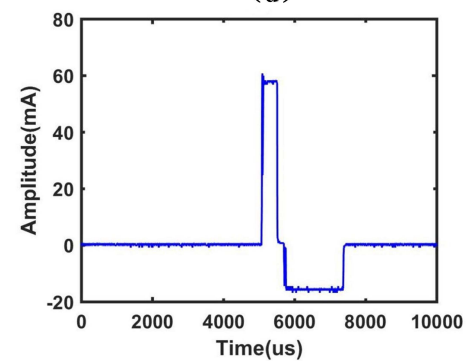

Fig. 5. Waveform of stimulation current with different parameters: (a) Amplitude$30 \mathrm{~mA}$, pulse- $400 \mu \mathrm{s}$, frequency- $50 \mathrm{~Hz}$; (b) Amplitude- $60 \mathrm{~mA}$, pulse- $400 \mu \mathrm{s}$, frequency-

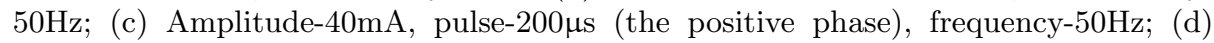
Amplitude-60mA, pulse- $400 \mu$ s (the positive phase), frequency-50Hz. (a) and (b) are symmetrical bipolar square wave. (c)-(d) are asymmetrical bipolar square wave.

\subsection{EMG-driven FES Experiments}

Preliminary experiment was conducted to verify the performance of the sEMGdriven FES system developed in this study. A handgrip dynamometer (Biometrics Ltd, UK) was used to measure MVC force for the grasp of the subject. The experimental procedure was as follows. Firstly, the subject was asked to perform maximum MVC handgrip contractions for five times using his left hand with EMG signals of FCU stored. Two silica gel electrodes were placed on the belly 
of FCU and another two were placed on the wrist for reference. Each contraction was kept for $5 \mathrm{~s}$ with $20 \mathrm{~s}$ rest between two consecutive contractions. For every $5 \mathrm{~s}$ contraction EMG signals, the data of the first second and the last second were discarded and the rest $3 \mathrm{~s}$ signals were used for EMG root mean square (RMS) value calculation for one contraction. The mean value of the RMS for the five contractions was set as $R M S_{\text {left }}$; Secondly, the electrodes were moved and placed on the same position on the right hand, then the subject performed the handgrip with his right hand and controlled the grip force in realtime according to the visual feedback of the force value displayed on a computer screen in front of him and the EMG RMS was calculated every $300 \mathrm{~ms}$ to map the FES intensity. Equ.6 shows the mapping relationship between sEMG and FES.

$$
\left\{\begin{array}{l}
Q=\frac{R M S_{\text {left }}-R M S_{\text {right }}}{R M S_{\text {left }}} \\
I_{R}=\frac{I_{\max }}{Q_{\max }-Q_{\min }} Q-\frac{I_{\max } Q_{\min }}{Q_{\max }-Q_{\min }}
\end{array}\right.
$$

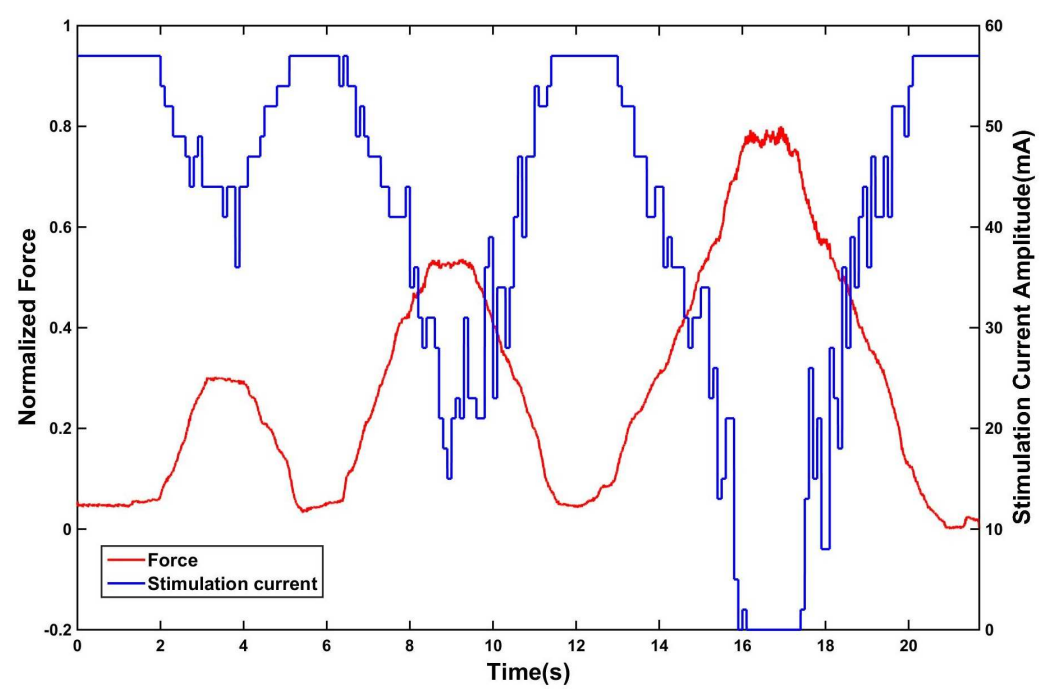

Fig. 6. The realtime FES output measurement based on the FCU EMG feedback when grip force varied. $Q_{\max }$ and $Q_{\min }$ were $100 \%$ and $20 \%$ respectively, $I_{\max }$ was set as 57 $\mathrm{mA}$. The three peaks of the force line represent $\mathrm{Q}=70 \%, \mathrm{Q}=40 \%$ and $\mathrm{Q}=20 \%$ in order respectively. When $Q>20 \%$, the FES current amplitude changed from $0 \mathrm{~mA}$ to $I_{\max }$ and be negative correlation with force. When $Q<20 \%$, the amplitude of the current is $0 \mathrm{~mA}$.

Fig. 6 shows the result of the experiment. It indicated that the FES intensity can be well controlled according to the EMG feedback in real time. The weaker the EMG is, the higher the FES intensity will be. 


\section{Conclusion}

This paper presents a multi-channel EMG-driven FES integration system for stroke rehabilitation. The designed system has advantages of wireless communication, multiple channels, portable size and real-time capability. On one hand, the system can be used as sEMG acquisition device and FES device respectively; on the other hand, the FES can be optimized by the EMG, which demonstrates the potential of driving the FES treatment process using the patients' muscle state and movement intention for stroke rehabilitation.

Acknowledgments. This work is supported by the National Natural Science Foundation of China (No. 51575338, 51575407, 51475427) and the Fundamental Research Funds for the Central Universities (17JCYB03).

\section{References}

1. Lynch, C.L., Popovic, M.R.: Functional electrical stimulation. IEEE control systems 28(2), 40-50 (2008)

2. Liberson, W.: Functional electrotherapy: stimulation of the peroneal nerve synchronized with the swing phase of the gait of hemiplegic patients. Arch Phys Med Rehabil 42, 101 (1961)

3. Popović, D.B.: Advances in functional electrical stimulation (fes). Journal of Electromyography and Kinesiology 24(6), 795-802 (2014)

4. Lyons, G.M., Sinkjær, T., Burridge, J.H., Wilcox, D.J.: A review of portable fesbased neural orthoses for the correction of drop foot. IEEE Transactions on neural systems and rehabilitation engineering 10(4), 260-279 (2002)

5. Edgerton, V.R., Roy, R.R.: Robotic training and spinal cord plasticity. Brain research bulletin 78(1), 4-12 (2009)

6. Lotze, M., Braun, C., Birbaumer, N., Anders, S., Cohen, L.G.: Motor learning elicited by voluntary drive. Brain 126(4), 866-872 (2003)

7. Quandt, F., Hummel, F.C.: The influence of functional electrical stimulation on hand motor recovery in stroke patients: a review. Experimental \& translational stroke medicine 6(1), 9 (2014)

8. Hong, I.K., Choi, J.B., Lee, J.H.: Cortical changes after mental imagery training combined with electromyography-triggered electrical stimulation in patients with chronic stroke. Stroke 43(9), 2506-2509 (2012)

9. Fujiwara, T., Kasashima, Y., Honaga, K., Muraoka, Y., Tsuji, T., Osu, R., Hase, K., Masakado, Y., Liu, M.: Motor improvement and corticospinal modulation induced by hybrid assistive neuromuscular dynamic stimulation (hands) therapy in patients with chronic stroke. Neurorehabilitation and neural repair 23(2), 125-132 (2009)

10. Fang, Y., Zhu, X., Liu, H.: Development of a surface emg acquisition system with novel electrodes configuration and signal representation. In: International Conference on Intelligent Robotics and Applications. pp. 405-414. Springer (2013)

11. Forvi, E., Bedoni, M., Carabalona, R., Soncini, M., Mazzoleni, P., Rizzo, F., OMahony, C., Morasso, C., Cassarà, D.G., Gramatica, F.: Preliminary technological assessment of microneedles-based dry electrodes for biopotential monitoring in clinical examinations. Sensors and Actuators A: Physical 180, 177-186 (2012) 